\title{
A Japanese Patient with Löfgren's Syndrome with an HLA-DR12 Allele and Review of Literature on Japanese Patients
}

\author{
Yuji Ishimatsu, ${ }^{1}$ Hanako Koyama, ${ }^{1}$ Masaomi Tomonaga, ${ }^{1}$ Tatsuhiko Harada, ${ }^{1}$ \\ Shota Nakashima, ${ }_{1}^{1}$ Atsuko Hara, ${ }^{1}$ Shintaro Hara, ${ }^{1}$ Tomoyuki Kakugawa, ${ }^{1}$ \\ Noriho Sakamoto, ${ }^{1}$ Tomayoshi Hayashi ${ }^{2}$ and Shigeru Kohno ${ }^{1}$
}

\author{
${ }^{1}$ Second Department of Internal Medicine, Nagasaki University School of Medicine, Nagasaki, Nagasaki, Japan \\ ${ }^{2}$ Department of Pathology, Nagasaki University Hospital, Nagasaki, Nagasaki, Japan
}

\begin{abstract}
Sarcoidosis is a granulomatous disorder of unknown etiology, with several clinical manifestations. Löfgren's syndrome is an acute type of sarcoidosis, characterized by the triad of arthritis, erythema nodosum, and bilateral hilar lymphadenopathy (BHL), which spontaneously resolve within about 2 years. Löfgren's syndrome is common among young white women from Nordic countries and Ireland, but it is very rare in Japan. Because the incidence of Löfgren's syndrome varies according to race, most studies on Löfgren's syndrome, including HLA typing, have been reported in Western countries. Indeed, HLA-DR3 has been reported to be associated with Löfgren's syndrome in Western countries, although the association between HLA typing and Japanese Löfgren's syndrome remains unclear. Here we present a Japanese patient with Löfgren's syndrome. A 34-year-old female patient was hospitalized with arthritis and erythema nodosum. Chest computed tomography revealed mediastinal and BHL. Endobronchial ultrasound-guided transbronchial needle aspiration showed non-caseating epithelioid cell granulomas. Löfgren's syndrome was thus diagnosed. Her ankle arthralgia and bilateral ankle swelling recovered without steroid treatment within two months, and the BHL almost completely diminished one year after admission. Her HLA genotype contains DR12. We also reviewed the literature on 11 Japanese patients with Löfgren's syndrome, showing that HLA-DR12 is present in five out of nine patients $(55.6 \%)$. The relevant data were unavailable in the remaining three patients. Importantly, only $5.4 \%$ of registered donors in the Japan Marrow Donor Program are positive for this allele. We suggest the potential link between HLA-DR12 and the pathogenesis of Löfgren's syndrome in Japanese patients.
\end{abstract}

Keywords: HLA typing; human leukocyte antigen; Japan; Löfgren's syndrome; sarcoidosis Tohoku J. Exp. Med., 2014 October, 234 (2), 137-141. C 2014 Tohoku University Medical Press

\section{Introduction}

Sarcoidosis is a systemic granulomatous disease that typically affects various organs such as the lungs, skin, eyes, and lymph nodes. The course and prognosis is associated with the mode of onset and the extent of the disease. Löfgren's syndrome is an acute type of sarcoidosis that manifests as a triad of arthralgia, erythema nodosum (EN), and bilateral hilar lymphadenopathy (BHL). The disease accounts for $20-30 \%$ of the cases of sarcoidosis in white individuals (ATS/ERS/WASOG Committee 1999). Löfgren's syndrome is reported to be more frequent in young white women (Grunewald and Eklund 2007), but it is very rare in Japan. Therefore, most studies on Löfgren's syndrome, including human leukocyte antigen (HLA) typing, have been reported form Western countries, while there is no review on the association between HLA typing and
Japanese Löfgren's syndrome. Herein, we report a case of Löfgren's syndrome in a Japanese woman. In this patient, endobronchial ultrasound-guided transbronchial needle aspiration (EBUS-TBNA) was useful in aiding diagnosis. HLA analysis revealed A22, B35, B44, DR12 and DR13 haplotypes. We searched the literature on Japanese Löfgren's syndrome and reviewed the clinical characteristics, including HLA haplotypes, of twelve Japanese patients with the complete triad of Löfgren's syndrome.

\section{Case Presentation}

The patient was a 34-year-old Japanese woman who had repeated remissions and exacerbations of arthralgia of the right ankle since December 2008. In August 2009, she developed myalgia of her lower body together with arthralgia. Subcutaneous induration with tenderness of the right lower leg was also observed. In September 2009, she

Received May 16, 2014; revised and accepted September 8, 2014. Published online September 30, 2014; doi: 10.1620/tjem.234.137.

Correspondence: Yuji Ishimatsu, M.D., Ph.D., Second Department of Internal Medicine, Nagasaki University School of Medicine, 1-7-1

Sakamoto, Nagasaki, Nagasaki 852-8501, Japan.

e-mail: yuji-i@nagasaki-u.ac.jp 


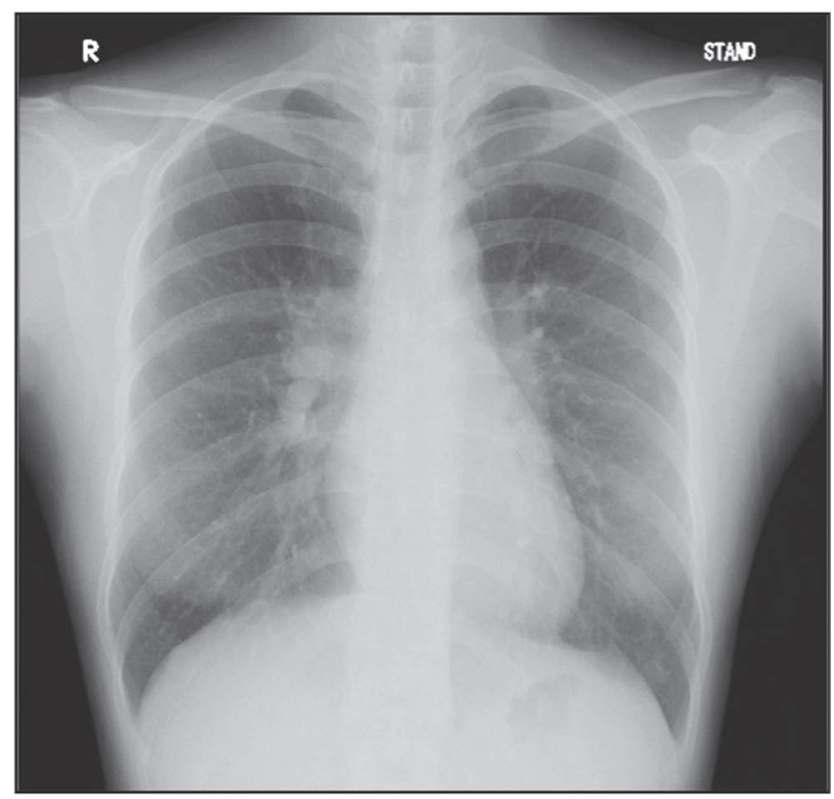

Fig. 1. Chest radiograph on admission.

Chest radiograph on admission showed bilateral hilar lymphadenopathy (BHL).

noticed occasional low-grade fever and visited the orthopedist. Since an extremity X-ray did not reveal distinct abnormalities, she was referred to our hospital for further examination. At that time, the ankle joint of her right leg was swollen and painful. No neurological and ophthalmological abnormalities were observed.

Physical examination revealed bilateral swelling of the ankles and EN of the right lower extremity, which was proved by biopsy. Her laboratory tests indicated a normal level of C-reactive protein (CRP) at $0.05 \mathrm{mg} / \mathrm{dL}$ (normal: $<0.17 \mathrm{mg} / \mathrm{dL}$ ), and slightly elevated level of soluble interleukin-2 receptor (sIL-2R) at $865 \mathrm{U} / \mathrm{mL}$ (normal: 145-518 $\mathrm{U} / \mathrm{mL}$ ). Angiotensin-converting enzyme (ACE) levels were within normal limits, at $17.5 \mathrm{U} / \mathrm{L}$ (normal: 7.0-25.0 U/L), and antinuclear antibody (ANA) and anti-cyclic citrullinated peptide (CCP) antibodies were negative. Respiratory function tests and arterial gases were normal. Electrocardiography and ultrasound cardiography showed normal findings. Her reaction to a tuberculin skin test was erythema of $11 \mathrm{~mm}$. HLA typing revealed HLA-A22, B35, B44, DR12, and DR13 haplotypes. Chest radiography revealed BHL (Fig. 1) and chest computed tomography (CT) scan demonstrated bilateral mediastinal and hilar lymphadenopathy (Fig. 2). Gallium scintigraphy showed abnormal radioisotope uptake by the hilar and pretracheal lymph nodes bilaterally. Bronchoalveolar lavage (BAL) fluid with $63.3 \%$ recovery rate showed a normal total cell count $\left(2.1 \times 10^{5}\right.$ cells $\left./ \mathrm{mL}\right)$, normal proportion of lymphocytes (6\%), and slight elevation of the $\mathrm{CD} 4 / \mathrm{CD} 8$ ratio (3.4). EBUS-TBNA revealed non-caseating epithelioid cell granulomas (Fig. 3), although transbronchial lung biopsy (TBLB) did not. Based on the above clinical presentation and diagnostic evaluations, she was diagnosed with Löfgren's syn-

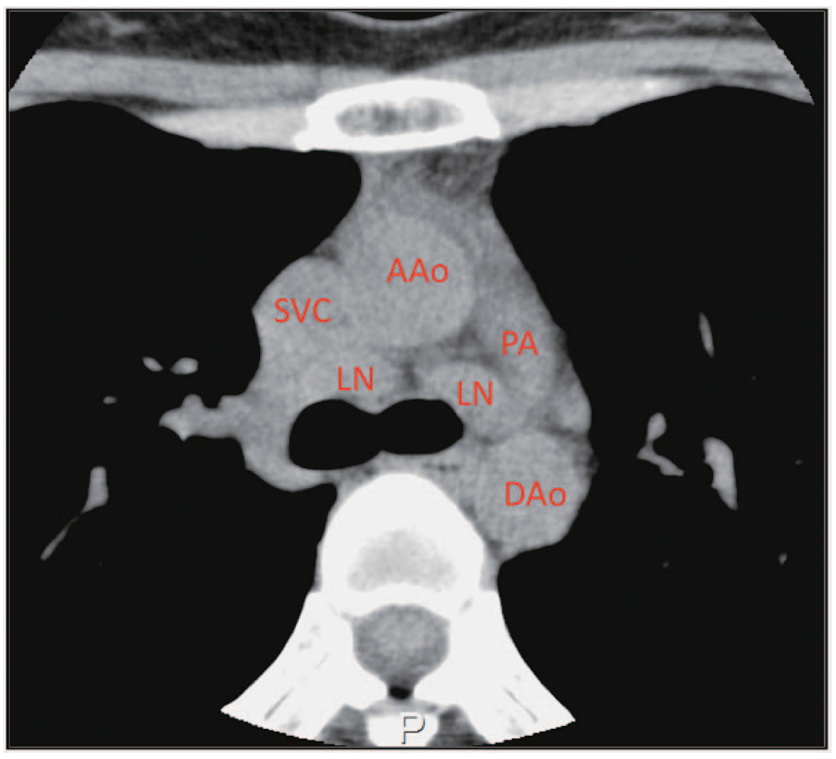

Fig. 2. Chest computed tomography (CT) scan on admission. Chest CT scan on admission showed bilateral hilar and mediastinal lymphadenopathy. Abbreviations: AAo, ascending aorta; DAo, descending aorta; LN, lymph node; PA, pulmonary artery; SVC, superior vena cava.

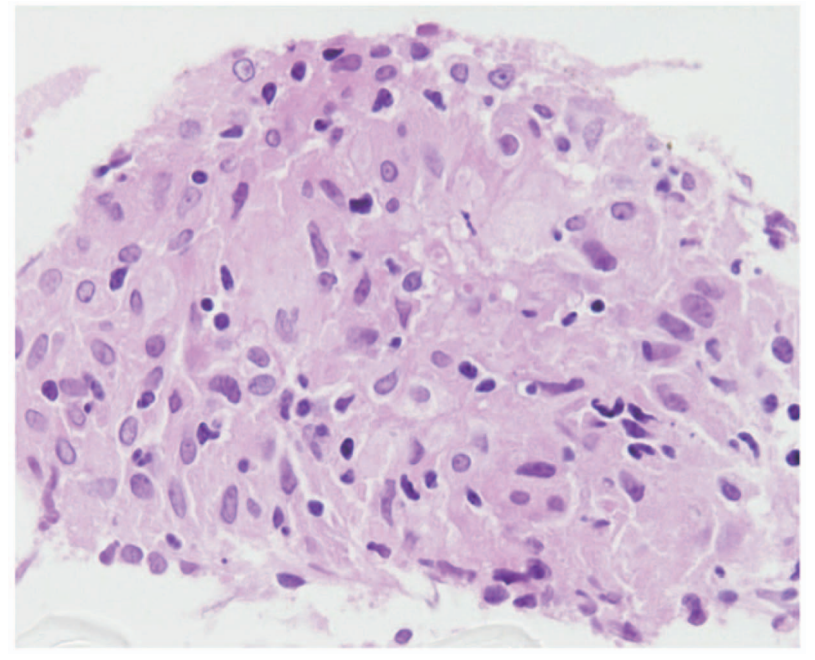

Fig. 3. Pathological finding of the mediastinal lymph node (hematoxylin-eosin staining).

A histological specimen obtained by Endobronchial ultrasound-guided transbronchial needle aspiration revealed non-caseating epithelioid cell granulomas.

drome. Her ankle arthralgia and bilateral ankle swelling recovered without treatment within 2 months, while the BHL almost completely diminished 1 year after admission.

\section{Discussion}

Sarcoidosis is a systemic granulomatous disease, which frequently presents with BHL, pulmonary infiltration, and ocular and skin lesions. Serum ACE is considered a possible biochemical marker of active sarcoidosis. 
Löfgren's syndrome, an acute type of sarcoidosis, has been reported to be more frequent in some geographical areas and races, particularly in young white women from the Nordic countries and Ireland, and has a favorable prognosis (Mana et al. 1999; Grunewald and Eklund 2007). Owing to its relatively common occurrence in Europe and the United States, a patient with Löfgren's syndrome presenting with the three characteristic symptoms can be diagnosed without biopsy in these countries (ATS/ERS/WASOG Committee 1999; Mana et al. 1999; Iannuzzi and Fontana 2011). In Japan, however, the occurrence of Löfgren's syndrome is extremely rare and Japanese clinicians likely underdiagnose and/or underreport this condition (Ohno and Ishigatsubo 2006). We searched the literature in English and in Japanese for Japanese patients who were diagnosed with Löfgren's syndrome with the confirmation of all three symptoms, arthritis, EN, and BHL, and reviewed the clinical characteristics of twelve patients, including our case (Table 1) (Iino et al. 1991; Koseki et al. 1998; Higashimoto et al. 2002; Niimi et al. 2003; Oshima et al. 2003; Izumo et al. 2005; Ohta et al. 2006; Sugimoto et al. 2006; Kosaka et al. 2007; Takasa et al. 2012; Mori et al. 2013). Ten patients shown in Table 1 were women $(83.3 \%)$, with a mean age of 34 years (range, 23-52 years), which was similar to the age range of patients in previous reports from Europe and the United States (Mana et al. 1999; Grunewald and Eklund 2007). The onset of disease was in March in four of the eight Japanese cases with known onset date $(50 \%)$. This supports the observations of previous reports that mentioned a seasonal clustering of Löfgren's syndrome in the spring months (Mana et al. 1999; Grunewald and Eklund 2007). Fever had developed in eight cases (75\%), which was high compared to $38 \%$ of 186 patients revisited by Mana et al. (1999). An increase in serum ACE levels was observed in only four of the twelve cases. The tuberculin skin test was negative in eight of the ten cases. Nine Japanese cases of Löfgren's syndrome were diagnosed histologically by fiberoptic bronchoscopy, video-assisted thoracoscopic surgery (VATS) and skin biopsy (for skin lesions other than EN), although these procedures are not considered necessary to diagnose Löfgren's syndrome in Europe and the United States. Our case was considered as stage I with BHL on chest radiography and was diagnosed as Löfgren's syndrome by EBUS-TBNA which histologically revealed non-caseating granulomas but TBLB not. Moreover, since Löfgren's syndrome is known to have an acute onset with remission of the sarcoidosis activity within the first 2 years (usually between 3 and 12 months), EBUSTBNA could be useful for the proper and prompt diagnosis of Löfgren's syndrome in a patient population in which its occurrence is uncommon, such as in Japan. BAL performed in ten patients showed that the median BAL lymphocytes percentage was $41 \%(6-66 \%)$ and the median CD4/CD8 ratio was 6.5 (1.1-19.9). Mana et al. (1999) reported that most patients with Löfgren's syndrome were treated with non-steroidal anti-inflammatory drugs for the relief of certain symptoms and that only 13 of 133 patients (9.8\%) required steroid treatment because the Löfgren's syndrome patients attained remission within the first 2 years. Grunewald and Eklund (2009) reported that 58 of 301 patients $(19 \%)$ required treatment with oral steroids at the time of or immediately after diagnosis and they suggested that DRB1*03-negative patients should be carefully observed and be considered for treatment at an early stage because only half of DRB $1 * 03$-negative patients recovered within 2 years although most DRB $1 * 03$-positive patients recovered. In our review of Japanese cases with Löfgren's syndrome, seven of 12 patients (58.3\%) were treated with oral steroids, and notably, all patients with HLA-DR12, except for our patient, were treated with oral steroids (Table 1). As these findings show, the proportion of Japanese patients with Löfgren's syndrome who receive oral steroids is higher than that in Western countries. It was unclear whether the increased use of oral steroids in Japan was influenced by the genetic association with the disease course or the unfamiliarity with Löfgren's syndrome in Japan.

The pathogenesis of sarcoidosis could be linked to a genetic predisposition and environmental factors. HLA class II alleles (HLA class II can form a complex with antigenic proteins and T-cell receptors to provide the first signal for T-cell activation) have been frequently reported as being involved in the development of sarcoidosis (Moller and Chen 2002), and several infectious agents (e.g., Propionibacterium acnes, Borrelia burgdorferi and Mycobacterium tuberculosis) have been implicated as potential causes of sarcoidosis (ATS/ERS/WASOG Committee 1999; Iannuzzi and Fontana 2011). Further, especially for Löfgren's syndrome, the greater prevalence in certain geographic areas and seasonal clustering during spring, as described previously, suggest that a combination of genetic predisposition and environmental factors are important in the development of this condition (ATS/ERS/ WASOG Committee 1999; Mana et al. 1999; Grunewald and Eklund 2007).

Several reports on HLA class II alleles associated with sarcoidosis and Löfgren's syndrome have shown variations according to region, race, and country. For example, the association with HLA-DR3 haplotype and Löfgren's syndrome has been widely reported in Europe and the United States (Grunewald and Eklund 2009; Grunewald et al. 2010). However, HLA-DR3 was not seen in any of the Japanese cases with Löfgren's syndrome described in Table 1 , while HLA-DR12 was present in five out of nine cases $(55.6 \%)$ (data were unavailable in the remaining three cases), which is high compared to the $5.4 \%$ rate of positivity seen in registered donors in the Japan Marrow Donor Program (JMDP) (Moriyama et al. 2006) and to the 22.2\% rate of Japanese patients with sarcoidosis (Ishihara et al. 1994). In 1994, Kunikane et al. reported the relationship between the pathogenesis of Japanese sarcoidosis and HLA-DRw52 (Kunikane et al. 1994), which is suggested as 


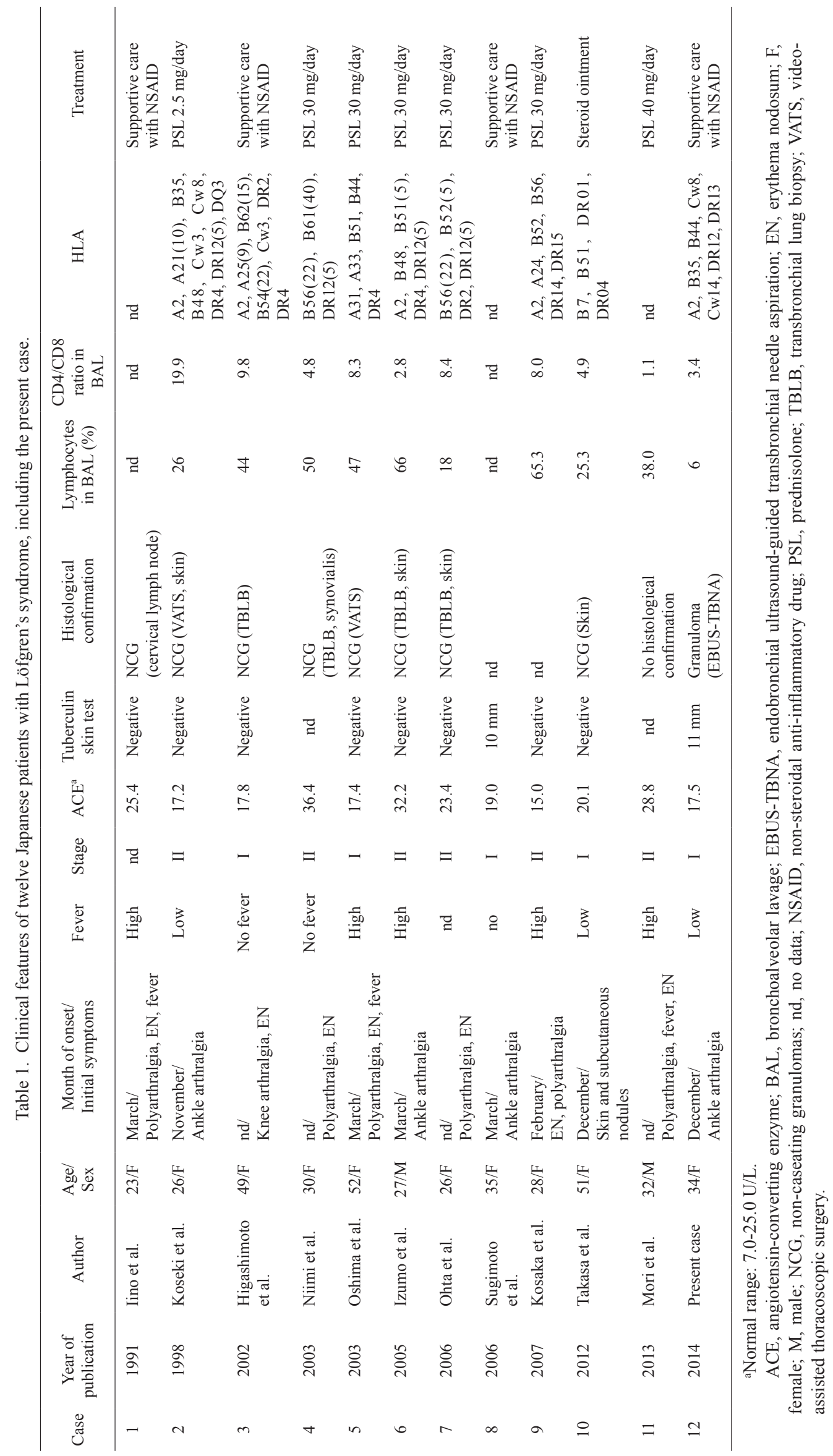


being due to linkage disequilibrium of DR5, comprising the DR12 and DR11 split antigens (Ina et al. 1989). Sato et al. (2010) investigated the clinical characteristics of sarcoidosis in terms of HLA class II genotypes in Japanese patients, and revealed that the HLA-DR12 allele was increased in Japanese patients with lung-predominant sarcoidosis compared with controls $(27.0 \%$ versus $6.5 \%, p<$ 0.0001), while HLA-DR12 allele was significantly increased in Scandinavian sarcoidosis patients with skin involvement (Darlington et al. 2014). These reports suggest that HLA-DR12 might play an important role in the pathogenesis of Japanese cases of sarcoidosis, especially in Löfgren's syndrome (as in our patient).

In conclusion, we report a case of Löfgren's syndrome in a Japanese woman with similar clinical characteristics to Western patients. However, our patient was found to have the HLA-DR12 allele, which is frequently found in Japanese patients with Löfgren's syndrome.

\section{Conflict of Interest}

The authors declare no conflict of interest.

\section{References}

American Thoracic Society (ATS), the European Respiratory Society (ERS) and the World Association of Sarcoidosis and Other Granulomatous Disorders (WASOG) Committee (1999) Statement on sarcoidosis. Am. J. Respir. Crit. Care Med., 160, 736-755.

Darlington, P., Gabrielsen, A., Sörensson, P., Tallstedt, L., Padyukov, L., Eklund, A. \& Grunewald, J. (2014) HLAalleles associated with increased risk for extra-pulmonary involvement in sarcoidosis. Tissue Antigens, 83, 267-272.

Grunewald, J., Brynedal, B., Darlington, P., Nisell, M., Cederlund, K., Hillert, J. \& Eklund, A. (2010) Different HLA-DRB1 allele distributions in distinct clinical subgroups of sarcoidosis patients. Respir. Res., 11, 25.

Grunewald, J. \& Eklund, A. (2007) Sex-specific manifestations of Löfgren's syndrome. Am. J. Respir. Crit. Care Med., 175, 40-44.

Grunewald, J. \& Eklund, A. (2009) Löfgren's syndrome: human leukocyte antigen strongly influences the disease course. $\mathrm{Am}$. J. Respir. Crit. Care Med., 179, 307-312.

Higashimoto, I., Arata, S.I. \& Niena, K. (2002) A case of acute sarcoidosis with polyarthralgia and erythema nodosum; Löfgren's syndrome. Nihon Kokyuki Gakkai Zasshi, 40, 679-682 (in Japanese).

Iannuzzi, M.C. \& Fontana, J.R. (2011) Sarcoidosis: clinical presentation, immunopathogenesis, and therapeutics. JAMA, 305, 391-399.

Iino, K., Muta, K., Sekiya, K., Umemura, T., Nishimura, J. \& Nawata, H. (1991) Löfgren's syndrome, acute sarcoidosis with polyarthralgia: a case report. Kyushu Rhumachi, 10, 148-151 (in Japanese).

Ina, Y., Takada, K., Yamamoto, M., Morishita, M., Senda, Y. \& Torii, Y. (1989) HLA and sarcoidosis in the Japanese. Chest, 95, 1257-1261.

Ishihara, M., Ohno, S., Ishida, T., Ando, H., Naruse, T., Nose, Y. \& Inoko, H. (1994) Molecular genetic studies of HLA class II alleles in sarcoidosis. Tissue Antigens, 43, 238-241.

Izumo, M., Sekiya, K., Sakai, T. \& Tojima, H. (2005) A male case of acute sarcoidosis with fever, polyarthralgia, erythema nodosum, and bilateral hilar lymphadenopathy: Löfgren's syndrome. Nihon Kokyuki Gakkai Zasshi, 43, 761-765 (in Japanese).

Kosaka, M., Aoki, M., Kawana, S., Tezuka, S., Takahashi, N., Yamaguchi, T. \& Munakata, K. (2007) A case of Löfgren's syndrome with acute aggravation of pulmonary lesion. Rinsho Hifuka, 61, 693-696 (in Japanese).

Koseki, Y., Terai, C., Nakajima, H., Tokuda, H., Ichikawa, N., Tsutsumino, M., Nakajima, A., Akama, H., Taniguchi, A., Hara, M. \& Kashiwazaki, S. (1998) A patient with acute sarcoidosis associated with fever, polyarthritis, and erythema nodosum: a typical case of Löfgren's syndrome. Ryumachi, 38, 23-28 (in Japanese).

Kunikane, H., Abe, S., Yamaguchi, E., Aparicio, J.M., Wakisaka, A., Yoshiki, T. \& Kawakami, Y. (1994) Analysis of restriction fragment length polymorphism for the HLA-DR gene in Japanese patients with sarcoidosis. Thorax, 49, 573-576.

Mana, J., Gomez-Vaquero, C., Montero, A., Salazar, A., Marcoval, J., Valverde, J., Manresa, F. \& Pujol, R. (1999) Löfgren's syndrome revisited: a study of 186 patients. Am. J. Med., 107, 240-245

Moller, D.R. \& Chen, E.S. (2002) Genetic basis of remitting sarcoidosis: Triumph of the trimolecular complex? Am. J. Respir. Cell Mol. Biol., 27, 391-395.

Mori, Y., Kikuchi, H., Ichikawa, H., Arakawa, Y., Maeta, T., Kohi, F. \& Awai, K. (2013) Two cases of Löfgren's syndrome: a literature-based comparison between Japanese and European Clinical Characteristics. Jpn. J. Sarcoidosis and other Granulomatous Disorders, 33, 91-96.

Moriyama, Y., Kato, K., Mura, T. \& Juji, T. (2006) Analysis of HLA gene frequencies and HLA haplotype for bone marrow donors in Japan (in Japanese). MHC, 12, 183-201 (in Japanese).

Niimi, T., Sato, S., Sugiura, Y., Akita, K., Maeda, H., Ninomiya, S., Achiwa, H., Masui, S., Bessho, Y., Maeno, K., Naniwa, T., Kotsuka, M., Morishita, M. \& Ueda, R. (2003) A case of sarcoidosis with rheumatic features (Löfgren's syndrome). Nihon Kokyuki Gakkai Zasshi, 41, 207-210 (in Japanese).

Ohno, S. \& Ishigatsubo, Y. (2006) The incidence of Löfgren's syndrome in Japanese: the number of patients affected, number of patients diagnosed and number of cases reported. Intern. Med., 45, 745-746.

Ohta, H., Tazawa, R., Nakamura, A., Kimura, Y., Maemondo, M., Kikuchi, T., Ebina, M. \& Nukiwa, T. (2006) Acute-onset sarcoidosis with erythema nodosum and polyarthralgia (Löfgren's syndrome) in Japan: a case report and a review of the literature. Intern. Med., 45, 659-662.

Oshima, M., Maeda, H., Furonaka, O., Doi, M., Nishizaka, T. \& Kuwabara, M. (2003) Sarcoidosis with multiple organ involvement emerging as Löfgren's syndrome. Intern. Med., 42, 534-537.

Sato, H., Woodhead, F.A., Ahmad, T., Grutters, J.C., Spagnolo, P., van den Bosch, J.M., Maier, L.A., Newman, L.S., Nagai, S., Izumi, T., Wells, A.U., du Bois, R.M. \& Welsh, K.I. (2010) Sarcoidosis HLA class II genotyping distinguishes differences of clinical phenotype across ethnic groups. Hum. Mol. Genet., 19, 4100-4011.

Sugimoto, T., Soumura, M., Hirata, K., Uzu, T., Nishino, Y. \& Kashiwagi, A. (2006) A case of Löfgren's syndrome, a variant of sarcoidosis, in a Japanese woman. Jpn. J. Intern. Med. (Naika Senmoni Kaishi), 18, 418-422.

Takasa, A., Nakayama, M., Bando, M., Mato, N., Nakaya, T., Hosono, T., Yamasawa, H. \& Sugiyama, Y. (2012) A case of nodular muscular sarcoidosis with Löfgren's syndrome. Jpn. J. Sarcoidosis and other Granulomatous Disorders, 32, 113-117. 